\title{
Journal of Mood Disorders and Therapy
}

ISSN: 2689-8969

Case Report

DOI: $10.36959 / 418 / 581$

\section{Audit of CBT for Depression and Anxiety in a Non- Randomized Routine-Practice Consecutive Case-Series of Adults with $\mathrm{ABI}$}

\author{
Brian Waldron*, Lisa Casserly and Aisling Lennon \\ Senior Clinical Psychologist and Clinical Neuropsychologist, Acquired Brain Injury Ireland, 13 Northumberland Avenue, \\ Dun Laoghaire, Ireland
}

\begin{abstract}
Introduction: There is a gap in the literature concerning the efficacy versus effectiveness of Cognitive Behavioral Therapy ( $C B T)$ for Acquired Brain Injury ( $A B I)$. Effectiveness relates to how treatments work in practice. Efficacy measures how they work in clinical trials with stringent inclusion and exclusion criteria. We assessed treatment in a regular clinic setting to determine effectiveness of CBT as it is usually delivered. This study audits the effectiveness of $\mathrm{CBT}$ in a real-world setting rather than efficacy.
\end{abstract}

Methods: A non-randomized routine-practice case-series of adults with ABI was used. We compared effect-sizes for 20 consecutive referrals, with various $A B I$ etiologies, to effect-sizes from previous $C B T$ outcome studies.

Results: Non-manualised individually-formulated and administered CBT had a positive effect. The effect-size was 1.74 for depression from pre-treatment to post-treatment, and 1.02 from pre-treatment to follow-up. There was an effect-size of 1.14 for anxiety from pre-treatment to post-treatment, but only 0.38 from pre-treatment to follow-up.

Conclusions: In our sample, CBT was effective from pre- to post-treatment, and there was evidence of long-lasting improvement over a 3.5 year follow-up for depression. While anxiety improved from pre- to post-treatment, there was substantial relapse over the 3.5 year follow-up. Implications for routine clinical practice are discussed.

\section{Keywords}

Cognitive behavioral therapy, Acquired brain injury, Case series audit, Treatment outcome, Depression and Anxiety

\section{Introduction}

CBT, based on a cognitive model of depression [1] conceptualizes thoughts as directly influencing the development and maintenance of emotions and behaviors [2]. CBT aims to teach cognitive skills [3] and focuses on challenging pessimism, promoting self-efficacy and improving mood. CBT typically includes behavioral aspects such as exposure to fears, activity-scheduling, behavioral-activation, relaxation, social-skills and assertiveness.

Mateer, Sira, and O'Connell [4] recommend "cognitive rehabilitation must integrate both cognitive and emotional interventions, and attend to belief systems about and affective responses to, cognitive challenges" (p. 62). Khan-Bourne and Brown [5] comment that "CBT has an intuitive appeal in the management of depression following brain injury (i) It accommodates and seeks to tackle the many personal and social sequelae that may contribute to psychological morbidity both acutely and chronically; (ii) It provides the therapist with a wide range of tools with which to work; and (iii) Is inherently flexible with the po- tential for accommodating differences in individual circumstances" (p. 98).

Reviews by Comper, Bisschop, Carnide, and Tricco [6] and Soo and Tate [7] revealed few RCTs for psychological interventions for anxiety or depression post-ABI. Gertler, Tate and Cameron [8] held off recommending any particular treatment based on their Cochrane review. However case and group-outcomes are reported on in meta-analyses by Stalder-Lüthy, et al. [9] and Waldron, Casserly, and

*Corresponding author: Dr. Brian Waldron, Senior Clinical Psychologist and Clinical Neuropsychologist, Acquired Brain Injury Ireland, 13 Northumberland Avenue, Dun Laoghaire, Ireland, Tel: 0035312804164

Accepted: January 02, 2020

Published online: January 04, 2020

Citation: Waldron B, Casserly L, Lennon A (2020) Audit of CBT for Depression and Anxiety in a Non-Randomized RoutinePractice Consecutive Case-Series of Adults with ABI. J Mood Disord Ther 2(1):32-35 
Citation: Waldron B, Casserly L, Lennon A (2020) Audit of CBT for Depression and Anxiety in a Non-Randomized Routine-Practice Consecutive Case-Series of Adults with ABI. J Mood Disord Ther 2(1):32-35

\section{O’Sullivan [10].}

Waldron, et al. [10] found that for depression, effectsizes ranged from 0 to 2.39 with an average effect-size of 1.15 (large effect). For anxiety, effect-sizes ranged from 0 to 3.47 with an average effect-size of 1.04 (large effect). By contrast, Stalder-Lüthy, et al. [9] found an overall effect-size of 0.69 suggesting "a medium effectiveness of psychological interventions" on depression (p. 1386).

This study (a) Determines the effectiveness of CBT in a routine sample and (b) Compares/audits this with efficacy as per the $C B T$ in $A B I$ literature.

\section{Materials and Methods}

\section{Participants}

Thirty-two consecutive referrals were used. Of the 32, 3 did not attend. Four attended once, stating they did not require intervention. Two couldn't engage due to cognitive problems. There are no pre-treatment scores for those 9 cases. This leaves 23 people for whom there was at least Time1 data. Of the 23, 4 dropped out. Unfortunately 3 drop-outs could not be included on an intention-to-treat (ITT) basis, as consent was sought retrospectively, and 3 dropouts did not consent. The ITT approach (post-treatment and follow-up data inputted as not changed) was used for 1 drop-out. ITT reduces the risk of falsely concluding CBT was effective by only using those who finish treatment. Scores were gathered prospectively as part of routine clinical practice at pre, post and follow-up. There was no blinding of outcome assessors.

All participants had ABIs including 12TBIs, 5 CVAs, 2 anoxic ABls and 1 tumour surgery. There were 12 men and 8 women with an average age of 39.45 (range 21 to 61). They had an average time since injury of 10.05 years (range 1 to 48 ). They had an average age at injury of 29.80 years (range 2 to 58 ).

Inclusion criteria for the organization, and therefore CBT, were (a) Between 18 and 65, (b) Without an active drug or alcohol problem, (c) Without a severe Axis 2 personality disorder, (c) With an ABI that is neither of chronic onset nor degenerative as per the Royal College of Physicians and British Society of Rehabilitation Medicine [11] definition. As this is a routine-practice study, many people had pre-ABI difficulties including mood and anxiety issues, previous drug and alcohol problems, marital problems, and histories of sexual or physical or emotional abuse in childhood. Some clients were expressing suicidal ideation at the time they began therapy.

\section{Instruments}

The Beck Depression Inventory - Second Edition (BDI-II) is a 21-item self-report scale [12]. Scores of 0-13 indicate minimal depression, scores of 14-19 indicate mild depression, scores of 20-28 indicate moderate depression and scores of 29-63 indicate severe depression. Cronbach's alpha of the total score was 0.92 for 500 outpatients and 0.93 for 120 college students. Test-retest reliability was 0.93 over a 1 week period for a subsample of 26 outpatients [12].

The Beck Anxiety Inventory (BAI) is a 21-item self-report scale [13]. Scores of 0-7 reflect minimal anxiety, scores of 8-15 indicate mild anxiety, whereas scores of 16-25 indicate moderate anxiety and scores of 26-63 indicate severe anxiety. The Cronbach's alpha of the total score was 0.92 for 160 outpatients reported on by Beck, Epstein, Brown, and Steer [14] and 0.94 for 40 outpatients reported on by Fydrich, Dowdall, and Chambless [15]. Test-retest reliability was reported to be 0.75 over a 1 week period for a subsample of 83 of the Beck, et al. [14] outpatients.

\section{CBT procedure}

Clients had one-to-one CBT with 45-60 minute sessions. CBT was not manualised. The average was 19 sessions with some clients having up to 30 sessions. CBT formulations were developed and shared as per models of PTSD [16]; Generalised Anxiety Disorder [17]; OCD [18]; panic disorder $[19,20]$ and depression [21]. Universal features included psycho-education on ABI. In addressing mood, CBT focused on the relationship between thinking, emotion and behaviors. A common technique was challenging thoughts using the 7-column thought-record and other approaches from Greenberger and Padesky [22]. Modification to CBT to account for cognitive issues has been addressed in a recent systematic review [23]. In our practice, modifications included reduction in the number of columns to 3 , evidence for, evidence against, and balanced thought. In relation to anxiety, discussion and behavioral experiments were used to tackle avoidance. The supervisor was a Senior Clinical Psychologist with 7 years' experience (including 3 years of Doctorate training) that was a Chartered Clinical Psychologist with The Psychological Society of Ireland and the British Psychological Society.

\section{Results}

Effect-sizes were calculated using $d=(M 1-M 2) / S D$. This formula is Glass's $\Delta$. According to Cohen [24] effect-size conventions are: small $=0.30$, medium $=0.50$, large $=0.80$.

The BDI-II $1 \times 3$ ANOVA $[F(2,55)=11.279, p<0.001]$ reached significance. Post-hoc Bonferroni tests revealed a significant difference between pre-treatment and post-treatment $[\mathrm{t}(38)=4.51, \mathrm{p}<0.001]$, and between pre-treatment and follow-up $[\mathrm{t}(36)=2.62, \mathrm{p}<0.05]$ indicating depression reduced significantly after CBT and remained statistically reduced at follow-up. There was an effect-size of 1.74 from pre-treatment to post-treatment (large effect) and 1.02 from pre-treatment to follow-up (large effect). Group means dropped from the moderate range at pre-treatment to the minimal range at post-treatment but had increased to the mild range at follow-up. The BDI-II manual indicates a cut-off of 17 for research. The group mean remained below this cutoff both at post-treatment and follow-up (Table 1).

The BAI $1 \times 3$ ANOVA $[F(2,54)=4.470, p<0.05]$ reached significance. Post-hoc Bonferroni tests revealed a significant difference between pre-treatment and post-treatment $[\mathrm{t}(38)=3.00, \mathrm{p}<0.01]$, but not between pre-treatment and follow-up $[\mathrm{t}(35)=1.08, \mathrm{p}>0.05]$ indicating anxiety reduced significantly after CBT but did not remain statistically reduced at follow-up. There was an effect-size of 
Citation: Waldron B, Casserly L, Lennon A (2020) Audit of CBT for Depression and Anxiety in a Non-Randomized Routine-Practice Consecutive Case-Series of Adults with ABI. J Mood Disord Ther 2(1):32-35

Table 1: Internal reliability, means, standard deviations, F values, effect-sizes and qualitative interpretations for the BDI-II and BAI scores.

\begin{tabular}{|l|l|l|l|l|l|l|l|l|l|l|l|}
\hline Measure & $\boldsymbol{\alpha}$ & $\begin{array}{l}\text { Pre-treatment } \\
\mathbf{N = 2 0} \\
\text { Mean }\end{array}$ & SD & $\begin{array}{l}\text { Post-treatment } \\
\mathbf{N = 2 0} \\
\text { Mean }\end{array}$ & SD & $\begin{array}{l}\text { Follow-up } \\
\text { N=18 } \\
\text { Mean }\end{array}$ & SD & F value & $\begin{array}{l}\text { Glass's } \Delta \\
\text { T1-T2 }\end{array}$ & Glass's $\Delta$ \\
T1-T3
\end{tabular}

BDI-II = Beck Depression Inventory - II; BAI = Beck Anxiety Inventory; a = Post-hoc Bonferroni comparison $(\mathrm{P}<0.05)$ : Pre-treatment versus post-treatment; $b=$ Post-hoc Bonferroni comparison $(P<0.05)$ : Pre-treatment versus follow-up; $\alpha=$ Cronbach's Alpha reliability at pretreatment; $\Delta=$ Glass's Delta effect-size; ${ }^{*} \mathrm{p}<0.05 ;{ }^{* *} \mathrm{p}<0.01 ;{ }^{* * *} \mathrm{p}<0.001$.

1.14 from pre-treatment to post-treatment (large effect) and 0.38 from pre-treatment to follow-up (small effect). Mean scores dropped from moderate at pre-treatment to mild at post-treatment but increased to the very upper end of the mild range at follow-up.

\section{Discussion}

The effect-sizes from pre to post-treatment in this effectiveness study compare favourably to effect-sizes reported on in previous meta-analyses $[9,10]$ of mainly efficacy studies (frequent reports of manualised treatment and a set number of sessions). Our results suggest the effectiveness of CBT (with a formulation and flexible treatment plan instead of manualised treatment or preset number of sessions) compares well with efficacy outcomes. The BDI-II manual indicates a cut-off score of 17 for research. At pre-treatment $75 \%$ were above the cut-off. At post-treatment $15 \%$ were above the cut-off. At follow-up $50 \%$ were above the cut-off. The overall group mean was below the cut-off at post-treatment and just about below the cut-off at follow-up. By contrast, while there were statistically and clinically significant improvements on the BAI from pre-treatment to post-treatment, there was a worsening of BAI scores at follow-up. In our sample, the effects of CBT on self-reported anxiety were not very long lasting.

$A$ retrospective concern was our use of the BDI-II and $B A I$. While there are numerous precedents in the CBT in $A B I$ literature for these measures, a doubt remains as to whether they may give an inflated estimate of anxiety and depression post-ABI. In our (anecdotal) experience the BDI-II puts too much emphasis on the cognitive aspects of low mood for it to be entirely reliable in $A B I$. This is also reflected in some research in the literature [25]. The HADS [26] by contrast doesn't emphasize fatigue, problems with decision-making, concentration, eating or sleeping patterns all of which can be present for people with $A B I$ but who are not depressed.

Similarly, other authors such as Leyfer, Ruberg, and Woodruff-Borden [27] express concern about the BAI stating "analysis revealed that while the BAI had acceptable sensitivity, it did not have high specificity in detecting any anxiety disorder. Thus although elevated BAI scores may indicate the presence of pathological anxiety, a significant portion of those who achieve elevated scores will in reality not have an anxiety disorder" (p. 455).

With respect to generalization of findings such as maintenance of depression score outcomes at follow-up, a consideration is that all participants were service-users of the host organization, and had either a residential or community service prior to, during and after CBT. The overall service received by participants was broader than purely $\mathrm{CBT}$. While this would be the case in virtually any $\mathrm{ABI}$ rehabilitation context where CBT is used, this is rarely explicitly acknowledged in the existing research literature.

While remaining below the research cut-off at follow-up, BDI-II mean scores worsened from the minimal range to the mild range over the follow-up. Additionally the BAl score at follow-up was not statistically different from pre-treatment. Taken together these statistical and clinical changes indicate a degree of relapse that occurs during the years after conclusion of CBT. Single-case-studies in $A B I$ have documented maintenance of CBT benefits at follow-up timescales of 1 to 6 months [28-31]. Group studies in ABI have shown similar maintenance of benefits, where the clinical range in which a group mean score lay, remained unchanged at 1 to 12 month follow-up [32-35]. Our findings imply that over longer timespans services need to plan for a proportion of service-users accessing specialist Clinical Psychology services more than once or intermittently.

Following on from this there is the broader question of trying to shed light on why some participants relapsed over the years. While a number of authors have queried links between failure to profit from $C B T$ and severity of $A B I$, in our experience life-events such as bereavement, major changes such as progressing from residential services to lower support community rehabilitation, or by contrast feeling stuck in residential services had roles. By contrast, many did well. On reflection we believe several factors contributed. Firstly, sharing the CBT formulation of predisposing, precipitating, maintaining and protective factors with clients was essential. This helped ground the therapy in a context that was broader than $A B I$ and brought into discussion pre-ABI predisposing factors and vulnerabilities to mood and anxiety problems occurring post-ABI. Secondly, psycho-education on the nature of the individual's $A B I$ was important as details given in acute and post-acute hospitals tended to be forgotten. Thirdly, not having a finite number of sessions, with the potential for a large number of sessions if necessary, gave scope to the ther- 
apists to develop a genuine client-centered relationship [36] including dealing with cases of bereavement and childhood abuse.

\section{Acknowledgements}

The authors would like to thank the 20 clients of $A B I$ Ireland who consented to have their information used as part of this study. The authors would also like to acknowledge the trust placed in our organization by the Irish Health Service Executive who funds our ongoing work nationally.

\section{References}

1. Beck AT (1967) Depression: Clinical, experimental, and theoretical aspects. Harper \& Row, New York.

2. Heimberg RG (2002) Cognitive-behavioral therapy for social anxiety disorder: Current status and future directions. Biol Psychiatry 51: 101-108.

3. Clark DA, Beck AT, Alford BA (1999) Scientific foundations of cognitive theory and therapy of depression. John Wiley \& Sons, New York.

4. Mateer CA, Sira CS, O'Connell ME (2005) Putting Humpty Dumpty together again: The importance of integrating cognitive and emotional interventions. J Head Trauma Rehabil 20: 62-75.

5. Khan-Bourne N, Brown RG (2003) Cognitive behaviour therapy for the treatment of depression in individuals with brain injury. Neuropsychol Rehabil 13: 89-107.

6. Comper P, Bisschop SM, Carnide N, et al. (2005) A systematic review of treatments for mild traumatic brain injury. Brain Inj 19: 863-880.

7. Soo C, Tate R (2007) Psychological treatment for anxiety in people with traumatic brain injury. Cochrane Database of Systematic Reviews.

8. Gertler P, Tate RL, Cameron ID (2015) Non-pharmacological interventions for depression in adults and children with traumatic brain injury. Cochrane Database of Syst Rev.

9. Stalder-Lüthy F, Messerli-Bürgy N, Hofer H, et al. (2013) Effect of psychological interventions on depressive symptoms in longterm rehabilitation after an acquired brain injury: A systematic review and meta-analysis. Arch Phys Med Rehabil 94: 1386-1397.

10. Waldron BA, Casserly LM, O'Sullivan C (2013) Cognitive behavioural therapy for depression and anxiety in adults with acquired brain injury. What works for whom? Neuropsychol Rehabil 23: 64-101.

11. Royal College of Physicians and British Society of Rehabilitation Medicine (2003) In: Turner-Stokes L, Rehabilitation following acquired brain injury: national clinical guidelines, RCP \& BSRM, London.

12. Beck AT, Steer RA, Brown GK (1996) Manual for the beck depression inventory II (BDI-II). The psychological corporation San Antonio, TX.

13. Beck AT, Steer RA (1993) Manual for the beck anxiety inventory (BAI). The psychological corporation, San Antonio, TX.

14. BBeck AT, Epstein N, Brown G, et al. (1988) An inventory for measuring clinical anxiety: Psychometric Properties. J Consult Clin Psychol 56: 893-897.

15. Fydrich T, Dowdall D, Chambless DL (1990) Aspects of reliability and validity for the Beck Anxiety Inventory. Paper presented at the National Conference on Phobias and Related Anxiety Disorders, Bethesda, MD.
16. Ehlers A, Clarke DM (2000) A cognitive model of posttraumatic stress disorder. Behav Res Ther 38: 319-345.

17. Wells A (1997a) Generalised anxiety disorder. In cognitive therapy of anxiety disorders: A practice manual and conceptual guide. Chichester: Wiley, 200-235.

18. Wells A (1997b) Obsessive compulsive disorder. In cognitive therapy of anxiety disorders: A practice manual and conceptual guide. Chichester: Wiley, 236-264.

19. Clark DM (1986) A cognitive approach to panic: Behav Res Ther. 24: 461-470.

20. Salkovskis PM, Clark DM (1990) Affective responses to hyperventilation: A test of the cognitive model of panic. Behaviour Research and Therapy 28: 51-61.

21. Beck AT, Rush AJ, Shaw BF, et al. (1979) Cognitive therapy of depression. Guilford Press, New York.

22. Greenberger D, Padesky CA (1995) Mind over mood: A cognitive therapy treatment manual for clients, Guilford Press, New York.

23. Gallagher M, McLeod HJ, McMillan TM (2016) A systematic review of recommended modifications of $C B T$ for people with cognitive impairments following brain injury. Neuropsychol Rehabil 29: 1-21.

24. Cohen J (1988) Statistical power analysis for the behavioral sciences. Lawrence Erlbaum Associates, Hillsdale, NJ.

25. Sliwinski M, Gordon WA, Bogdany J (1998) The beck depression inventory: Is it a suitable measure of depression for individuals with traumatic brain injury? J Head Trauma Rehabil 13: 40-46.

26. Zigmond AS, Snaith RP (1983) The hospital anxiety and depression scale. Acta Psychiatr Scand 67: 361-370.

27. Leyfer OT, Ruberg JL, Woodruff-Borden J (2006) Examination of the utility of the Beck Anxiety Inventory and its factors as a screener for anxiety disorders. Anxiety Disorders 20: 444-458.

28. Arco L (2008) Neurobehavioural treatment for obsessive-compulsive disorder in an adult with traumatic brain injury. Neuropsychol Rehabil 18: 109-124.

29. Kneebone II, Hull SL (2009) Cognitive behaviour therapy for post-traumatic stress symptoms in the context of hydrocephalus: A single-case. Neuropsychol Rehabil 19: 86-97.

30. Lincoln NB, Flannaghan T (2003) Cognitive behavioral psychotherapy for depression following stroke: A randomized controlled trial. Stroke 34: 111-115.

31. McMillan TM (1991) Post-traumatic stress disorder and severe head injury. British Journal of Psychiatry 159: 431-433.

32. Arundine AM, Bradbury CL P, Dupuis KM A, et al. (2012) Cognitive behavior therapy after acquired brain injury: Maintenance of therapeutic benefits at 6 months post treatment. J Head Trauma Rehabil 27: 104-112.

33. Bradbury CL, Christensen BK, Lau MA, et al. (2008) The efficacy of cognitive behavior therapy in the treatment of emotional distress after acquired brain injury: Arch Phys Med Rehabil 89: S61-S68.

34. Bryant RA, Moulds M, Guthrie R, et al. (2003) Treating acute stress disorder following mild traumatic brain injury. Am J Psychiatry 160: 585-587.

35. Hodgson J, McDonald S, Tate R, et al. (2005) A randomised controlled trial of a cognitive-behavioural therapy program for managing social anxiety after acquired brain injury. Brain Impairment 6: 169-180.

36. Rogers CR (1961) On becoming a person: A therapist's view of psychotherapy. Houghton Mifflin Company, Boston. 